\title{
Herbicide Sulcotrione
}

\author{
Nanxiang $\mathrm{Wu}$ \\ Department of Environmental Health, Institute of hygiene \\ Zhejiang Academy of medical Sciences \\ P.R.China
}

\section{Introduction}

Sulcotrione is a new kind of triketone-type herbicide, which inhibits the activity of plant 4hydroxyphenylpyruvate dioxygenase (HPPD) [1]. It has been widely applied to control and prevent wild grass and weeds for crops such as barley, wheat and maize in Europe, the USA and many other countries, including China, since 2000 [2]. HPPD also exists in mammals for the catabolism of tyrosine. Although sulcotrione shows low toxicity in subchronic and chronic toxicity testing, it can also, through the food chain, be continuously transferred to and accumulate in the human body when it is widely used and applied and when part of its residue in the soil is absorbed by and aggregated in plants. Another part of its residue pollutes the water table through surface runoff and the underground permeable layer. Therefore, sulcotrione, as an HPPD inhibitor, has potential risks to human health, and its possible role as an environmental pollutant must raise attention and vigilance.

The residual level of sulcotrione in the soil directly affects its accumulation in agricultural products and is closely related to the level of human exposure. Currently, there is a standard for sulcotrione residue in the soil. Therefore, the investigation of the sulcotrione soil residual levels can provide scientific evidence for the rational application of sulcotrione and the establishment of pesticide residue standards. In addition, this investigation also can supply more data for soil pesticide monitoring databases and provide an informative source for scientific research.

HPPD, a target molecule of sulcotrione, exists universally in prokaryotes and eukaryotes. The catabolism of tyrosine is repressed when HPPD is inhibited by sulcotrione. Epinephrine is derived from tyrosine. The detection of tyrosine and epinephrine levels in the blood of rats exposed to sulcotrione can reflect the inhibiting effect of HPPD on epinephrine levels. The regulatory mechanisms for blood glucose are complicated. There are many possible pathways for sulcotrione to interfere with blood glucose levels. The rats were exposed to different doses of sulcotrione for different times to get blood glucose levels, enzyme activity, hormone levels, etc. The correlations between blood glucose levels and different parameters were analyzed in the rats exposed to sulcotrione to establish or exclude the possible interference of sulcotrione with blood glucose regulation.

In vitro experiments have already shown that sulcotrione can specifically, effectively and reversibly inhibit hepatic HPPD activity. However, there are few in vivo reports on this question. In this study, we investigate, the effects of sulcotrione on hepatic enzyme activity, tyrosinemia and cornea damage through subacute and chronic toxicity tests in rats. 


\section{Materials and methods}

\subsection{The investigation of sulcotrione soil residual levels}

\subsubsection{Soil sample collection}

The soil samples were collected following the sampling rules of national farmland and the environmental standards for safety, high quality and pollution-free agricultural products GB/T18047.1-2001 from September 2007 to September 2008 within eight regions in the Zhejiang Province, P.R. China. The soil sampling depth was 0 to $20 \mathrm{~cm}$. A single soil sample was a mixture of multiple (5) spots. A total of 200 soil samples were collected. At the same time, a total of 10 samples (from a mixture of 10 different regions) of cultured plants (corn) were collected in Quzhou and Dongyang in the Zhejiang Province.

\subsubsection{Sample preparation}

One gram samples (air-dried and passed through a 100 mesh sieve) were dissolved in $1 \mathrm{~mL}$ of methanol by mixing for 30 seconds. After incubation for 30 minutes, the sample was treated with ultrasound for 10 minutes. Then the sample was centrifuged for 5 minutes at $29000 \mathrm{~g}$ and the supernatant was collected and filtered through a membrane for detection.

\subsection{Sulcotrione analysis}

\subsubsection{HPLC chromatographic conditions [3-4]}

The mobile phase consisted of methanol:0.1M sodium dihydrogen phosphate triethylamine $=40: 60(\mathrm{v} / \mathrm{v})$. The flow rate was $0.8 \mathrm{~mL} / \mathrm{min}$ (A pump: $0.1 \mathrm{M}$ sodium dihydrogen phosphate triethylamine buffer $0.48 \mathrm{~mL} / \mathrm{min}, \mathrm{B}$ pumb: methanol $0.32 \mathrm{~mL} / \mathrm{min}$ ). The detection wavelength was $254 \mathrm{~nm}$. The column temperature was room temperature. The sensitivity: was 0.001 AUFS. The injection volume was $10 \mu l$.

\subsubsection{Sulcotrione standard curve}

\subsubsection{Preparation of the standard buffer}

A $0.0100 \mathrm{~g}$ (accuracy to $0.0001 \mathrm{~g}$ ) sulcotrione standard sample was accurately weighed. It was dissolved in a small volume of methanol which was poured into a $100 \mathrm{~mL}$ volumetric flask. It was diluted in methanol to the final scale. It was the standard stock sulcotrione solution. Before use, the stock solution was diluted in methanol to $10 \mathrm{mg} / \mathrm{L}$ as the working solution. The sulcotrione standard HPLC profile was made according to the above mentioned chromatographic conditions. The sulcotrione standard analysis sample was provided by Sigma and had a purity of more than $98 \%$ (the number is 46318 , which is valid until March 2013).

\subsubsection{Calculating the results}

The sample analytic results were extrapolated based on the area external standard method. The concentration calculation was: $X=$ solution volume $x C$ /sample weight, where $X$ is the sulcotrione concentration $(\mathrm{mg} / \mathrm{kg})$ and $\mathrm{C}$ is the concentration calculated from the standard curve.

\subsubsection{Statistical analysis}

SPSS 15.0 version was used for the statistical analysis. The results are presented as mean \pm SD. SD is the standard deviation. 


\subsection{Investigation of tyrosine levels in a population who might have had exposure to sulcotrione.}

\subsubsection{Determining the population}

The population consisted of forty males and forty females (80 totals) who work in the sulcotrione industry and another forty males and forty females who do not work in the sulcotrione industry; ten laboratory staff who have had contact with sulcotrione and 10 staff who do not have had contact were also included.

\subsubsection{Blood collection.}

The blood was centrifuged at $3000 \mathrm{~g}$ for 15 minutes. Then the supernatant was collected and cryopreserved at $-18^{\circ} \mathrm{C}$. The blood sample was prepared for sulcotrione detection as follows: The frozen serum was thawed at room temperature. A certain volume of the serum sample was transferred and mixed with the same volume of methanol. After this, it was treated with ultrasound for 10 minutes. And then the sample was placed on table for 10 minutes. After centrifuging at $29000 \mathrm{~g}$ for 5 minutes, the supernatant was passed through the membrane for detection. The blood sample was prepared for tyrosine detection as follows: a certain volume of sample was dissolved in the same volume of $0.59 \mathrm{M} \mathrm{HClO}_{4}$. It was mixed for 30 seconds and centrifuged at $29000 \mathrm{~g}$ for 5 minutes. The supernatant was collected and passed through the membrane for detection.

\subsubsection{Detection methods [5-7]}

\subsubsection{Standard materials}

The analytical standard sulcotrione was purchased from Sigma as described as 2.2.2.1. The analytical standard tyrosine was provided by Dikma and had purity $\geq 99.5 \%$ (the case No. is 0-110, which is valid until August of 2013).

The chromatographic conditions for tyrosine detection were: a mobile phase of acetonitrile:water = 5:95 (v/v); a $250 \mathrm{~mm} \times 4.6 \mathrm{~mm} \mathrm{C}_{18}$ column with particle size $5 \mu \mathrm{m}$; a flow rate of $0.8 \mathrm{~mL} / \mathrm{min}$; and a detection wavelength of $210 \mathrm{~nm}$. The chromatographic conditions for sulcotrione detection were: a mobile phase of methanol:0.1 $\mathrm{M}$ sodium dihydrogen phosphate and triethylamine buffer $=40: 60(\mathrm{v} / \mathrm{v})$; a $250 \mathrm{~mm} \times 4.6 \mathrm{~mm} \mathrm{C} 18$ column with particle size $5 \mu \mathrm{m}$; a flow rate of $0.8 \mathrm{~mL} / \mathrm{min}$; and a detection wavelength of $254 \mathrm{~nm}$. Tyrosine and sulcotrione were quantified using the extrapolation method. Under the chromatographic conditions suitable for instrument characteristics, tyrosine and sulcotrione were obtained in a linear correlation curve. The linear regression equation for sulcotrione was $y=20742.17 x$ +655.12 , with a correlation coefficient of 0.9993 . The linear regression equation for tyrosine was $y=207412.2 x-0.1092$, with a correlation coefficient of 0.9985 .

\subsubsection{The precision, accuracy and detection limit}

The detection precision, accuracy and detection limit for the sulcotrione and tyrosine detection methods were:

\begin{tabular}{|c|c|c|c|}
\hline parameters & Precision (RSD, \%) & $\begin{array}{c}\text { Accuracy } \\
\text { (recovery, \%) }\end{array}$ & Detection limit \\
\hline Sulcotrione & $4.83-6.79$ & $94.65-109.23$ & 0.044 mg.L-1 \\
\hline Tyrosine & $1.70-8.70$ & $89.63-108.22$ & 0.13 umol.L-1 \\
\hline
\end{tabular}

RSD: relative standard deviation. 
According to the requirements of the testing methodology, the relative standard deviation (RSD) is less than $10 \%$ and the recovery rate is between $90-110 \%$. The results show that HPLC method for sulcotrione and tyrosine detection meets the above requirement to ensure that the experimental data is accurate and reliable.

\subsubsection{Statistical analysis}

The statistical software SPSS15.0 was used for the one-way ANOVA analysis, least significant difference method (LSD) analysis and Dunnett's T test. The Pearson method is used for linear correlation analysis. The data are presented as mean \pm SD.

\subsection{The test of rats exposed to sulcotrione for 28 days to determine the time and response relationship between blood sulcotrione and tyrosine and main toxic response.}

\subsection{1 dose group [8-9]}

The original sulcotrione (purity $>95 \%$ ) was provided by a domestic corporation. The acceptable daily intake (ADI) of $0.005 \mathrm{mg} / \mathrm{kg}$, extrapolated from the results of a sulcotrione rat chronic toxicity test for no observed adverse effect level (NOAEL), was designated as the low-dose group; the medium-dose group was $0.05 \mathrm{mg} / \mathrm{kg}$ and the high-dose group was 0.5 $\mathrm{mg} / \mathrm{kg}$. A group without sulcotrione exposure was the control group. There were 28 rats in each group (equal numbers of males and females).

\subsubsection{Route of exposure, time and test indicators}

The rats were fed with sulcotrione dissolved in cooking oil once a day for 28 continuous days. The control group was fed cooking oil only. Blood was collected from the tail vein at days $0,7,14,21$, and 28 after sulcotrione exposure. The rats were not fed on the days of blood collection. At day 28, half the male and female rats were sacrificed and the sulcotrione feeding was discontinued for the remaining rats. At days 35 and 42, blood was collected from the tail vein. During the feeding, the body weight, diet, hair and activity of the rats were routinely checked and recorded. After they were sacrificed, the liver, kidneys, adrenal glands and other organs were collected for pathologic study. The organ and body weight ratio was calculated.

\subsubsection{The time of administration, sampling and sample preparation.}

Sulcotrione was given to rats at the same time each day (around 16:00). The rats were fasted from 21:00 the day before blood collections. The blood was collected at 8:00 the next day. The blood was centrifuged at $3000 \mathrm{~g}$ for 15 minutes. The serum was collected and stored at $18^{\circ} \mathrm{C}$ for cryopreservation. The blood sample was prepared for sulcotrione detection as follows. The serum was thawed at room temperature. An aliquot of serum was added to the same volume of methanol and mixed for 30 seconds. The mixture was treated with ultrasound for 10 minutes and placed on table for another 10 minutes. Then the sample was centrifuged at $29000 \mathrm{~g}$ for 5 minutes. The supernatant was collected and filtered through the membrane for detection. The blood sample was prepared for tyrosine detection as follows. The serum was thawed at room temperature. An aliquot of serum was added to the same volume of $0.59 \mathrm{M} \mathrm{HClO}_{4}$ and mixed for 30 seconds. The sample was centrifuged at $29000 \mathrm{~g}$ for 5 minutes. The supernatant was collected and filtered through the membrane for detection. Blood glucose was directly measured from tail vein blood sampling. 


\subsubsection{The analysis method and quality control of detection indicators}

The rat blood tyrosine and sulcotrione HPLC detection indicators were established. The accuracy, precision and detection limit for the detection method was under quality control (2.3.1). The blood glucose was detected by the Johnson \& Johnson Rapid Blood Glucose Detector (USA). The instrument was calibrated with standard reference liquid before blood glucose detection. The original sulcotrione has more than $98.9 \%$ active component. The peak area extrapolation method was used for quantitative analysis of tyrosine and sulcotrione.

\subsubsection{Statistical analysis}

The statistical software SPSS15.0 was used for one way ANOVA analysis, least significant difference method (LSD) analysis and Dunnett's T test. The Pearson method was used for linear correlation analysis. The data are presented as mean $\pm \mathrm{SD}$.

\subsection{Effects of sulcotrione on hepatic enzymes involved in tyrosine catabolism, tyrosinemia, and blood glucose in rat. \\ 2.5.1 Animals and treatments}

Sulcotrione with a purity of $>95.5 \% \mathrm{w} / \mathrm{w}$, was supplied by Jia Hua Import \& Export Co., Ltd (Zhejiang, China). male Alpk:APfSD (Wistar-derived) rats, aged from 5 to 6 weeks and obtained from Zhejiang Experimental Animal Center [SCXK (zhe) 2008-0033], were housed in stainless steel, wire bottom cages under standard housing conditions (controlled atmosphere with 12:12 h light/dark cycles, $55 \pm 5 \%$ humidity and an ambient temperature of $22 \pm 3^{\circ} \mathrm{C}$ ). The rats were fed on a commercial powdered diet (GB 14924-2001) and given filtered water ad libitum. Rats were acclimatized for 3 days prior to the experiment. Groups of 8 sulcotrione-treated rats and 8 control rats were dosed with either corn oil alone or sulcotrione in corn oil at $5 \mathrm{ml} / \mathrm{kg}$ body weight at $0.1,0.5$, and $5 \mathrm{mg} / \mathrm{kg} /$ day for 90 days. Throughout the study, clinical signs were observed, body weight was recorded weekly, and food consumption was monitored twice weekly. Animal care and monitoring were carried out in accordance with strict guidelines issued by the P.R. China legislation. All animal procedures and treatments were performed according to our Institute Animal Care and Use Committee (Certificate No. IACUC-03-001) and animals were terminated when deemed to be under moderate stress or discomfort.

\subsubsection{Hepatic enzymes involved in tyrosine catabolism assay}

The rats were sacrificed after the 90 days test by inhalation of an overdose of halothane as previously described. Livers were removed and then homogenized with 10 up-down strokes in $30 \mathrm{ml}$ of ice-cold $0.32 \mathrm{M}$ sucrose, and then diluted to give a $20 \%(\mathrm{w} / \mathrm{v})$ homogenate. The homogenate was then centrifuged at $105,000 \mathrm{~g}$ for $60 \mathrm{~min}$ at $4^{\circ} \mathrm{C}$ to remove particulate material. The supernatant (cytosol fraction) was stored in aliquots at $-70^{\circ} \mathrm{C}$ prior to the assay of activities of TAT, HPPD and HGO. The protein content in the supernatant was measured using bovine plasma albumin (BSA) as the internal standard. TAT was assayed in liver cytosol by the method of Schepartz, 1969. HPPD and HGO were measured in liver cytosol by monitoring oxygen consumption after addition of the relevant substrate by the methods described by Ellis et al., 1995, respectively.

\subsubsection{Serum epinephrine and tyrosine analysis}

After oral glucose tolerance test, femoral artery blood was adopted and then separated the serum. Serum epinephrine and tyrosine were measured by rat epinephrine ELISA kit 
(Uscnlife Science \& Technology Company, USA) and High Performance Liquid Chromatography (HPLC, SHIMADZU, LC-20AD), respectively.

\subsubsection{Fasting blood glucose test and Oral glucose tolerance test}

During treatment fasting blood glucose of tail vein in rat were measured in 0, 30, 60, 90 day (Fasting time: from 08:00 to 14:00) using fast blood glucose meter (Johnson \& Johnson Services Inc. USA). After final dosing, all rats were fasting for overnight (about 12 hours), and then oral glucose tolerance test was adopted. We first measured blood glucose of tail vein in rat in $0 \mathrm{~min}$, after that immediately giving glucose water solution $(2 \mathrm{~g} / \mathrm{kg} \mathrm{b.w})$, then blood glucose were measured in 30, 60, 120 min respectively.

\subsubsection{Statistical analysis}

The differences between sulcotrione-treated animals and controls were analyzed using SPSS Version 13.0 for Windows (SPSS Inc., Chicago, IL, USA). Changes in the concentration of tissue tyrosine or the enzymes involved in tyrosine catabolism in the time following sulcotrione treatment were analyzed using an analysis of variance followed by the Dunnett $t$ test. A p-value below 0.05 was considered to be statistically significant between experimental groups

\section{Results}

\subsection{Sulcotrione levels in the environment}

\subsubsection{Sulcotrione soil residual levels}

Sulcotrione soil residual levels in the area where the sulcotrione was used in Zhejiang Province are summarized in table1-1 and figure1-1. The mean sulcotrione soil residue was between $0.19-0.47 \mathrm{mg} / \mathrm{kg}$.

\begin{tabular}{|c|c|c|c|c|c|c|c|c|c|}
\hline area & Quzhou & Wenzhou & Jiande & Dongyang & Haining & Ninghai & Longyou & Jinhua & Total \\
\hline $\mathrm{N}$ & 37 & 20 & 24 & 34 & 10 & 10 & 28 & 37 & 200 \\
\hline $\begin{array}{c}\text { Mean } \pm \\
\text { sd }\end{array}$ & $0.30 \pm 0.18$ & $0.28 \pm 0.15$ & $\begin{array}{c}0.35 \pm \\
0.19\end{array}$ & $0.33 \pm 0.19$ & $\begin{array}{c}0.19 \pm \\
0.08\end{array}$ & $0.33 \pm 0.18$ & $0.41 \pm 0.19$ & $0.47 \pm 0.17$ & $0.35 \pm 0.19$ \\
\hline
\end{tabular}

Table 1.1. Sulcotrione soil residual levels from the sampling area in Zhejiang province $(\mathrm{mg} / \mathrm{kg})$

Figure 1-2 shows a plot of the distribution of the sulcotrione residual levels in the 200 soil samples against the expected normal probability distribution. The scatter graph follows an approximately straight line. So the test data follows a normal distribution. The arithmetic average of the sulcotrione soil residual levels in Zhejiang Province was $0.35 \pm 0.19 \mathrm{mg} / \mathrm{kg}$.

\subsubsection{Sulcotrione corn residual levels}

The sulcotrione residual levels in the 10 corn samples from the Quzhou and Dongyang areas are shown in Table 1-2. The detected values are between 0.02 to $0.10 \mathrm{mg} / \mathrm{kg}$. 


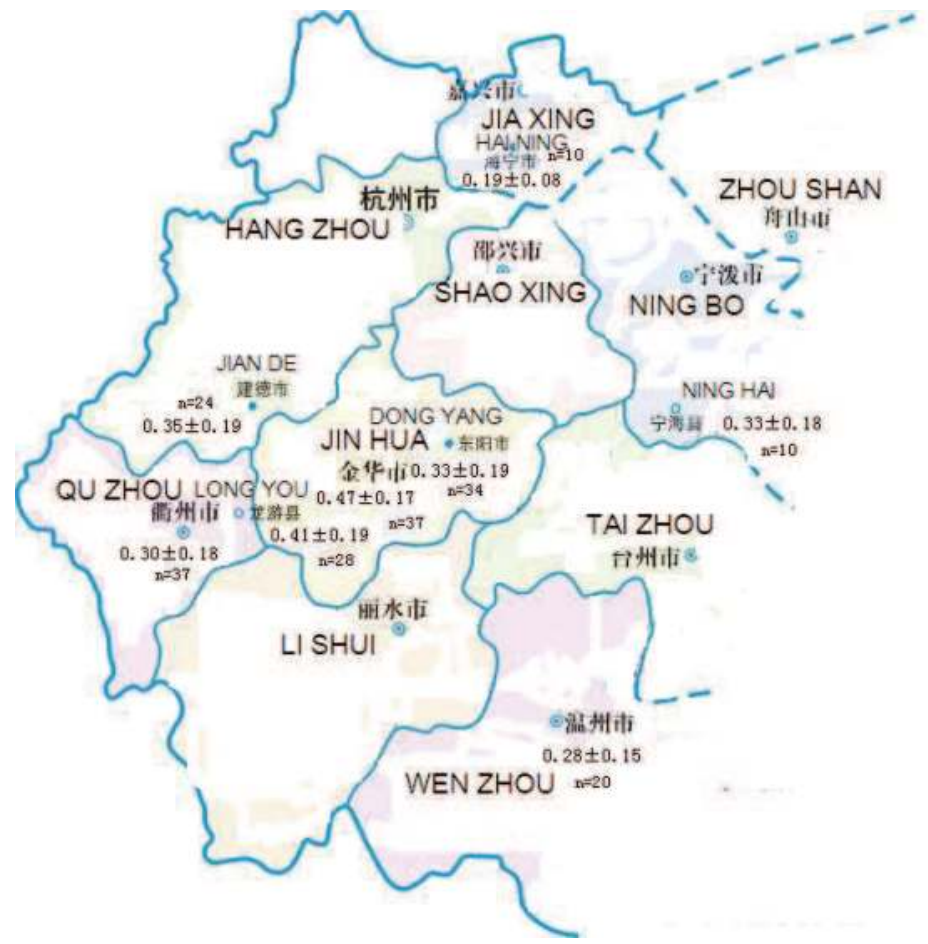

Fig. 1-1. The distribution of sulcotrione soil residual levels in Zhejiang Province $(\mathrm{mg} / \mathrm{kg}, \mathrm{n}$ : sample number)

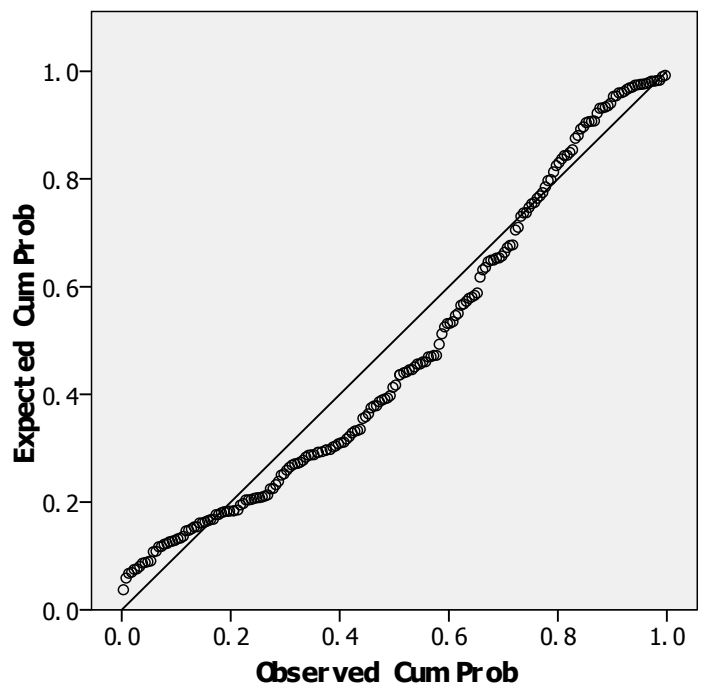

Fig. 1-2. The normal distribution P-P plot for sulcotrione soil residue. 


\begin{tabular}{|c|c|c|c|c|c|c|c|c|c|c|}
\hline $\begin{array}{c}\text { Sample } \\
\text { number }\end{array}$ & 1 & 2 & 3 & 4 & 5 & 6 & 7 & 8 & 9 & 10 \\
\hline Average & 0.03 & 0.06 & 0.05 & 0.04 & 0.02 & 0.08 & 0.07 & 0.10 & 0.05 & 0.07 \\
\hline
\end{tabular}

Table 1-2. Sulcotrione corn residual levels $(\mathrm{mg} / \mathrm{kg})$

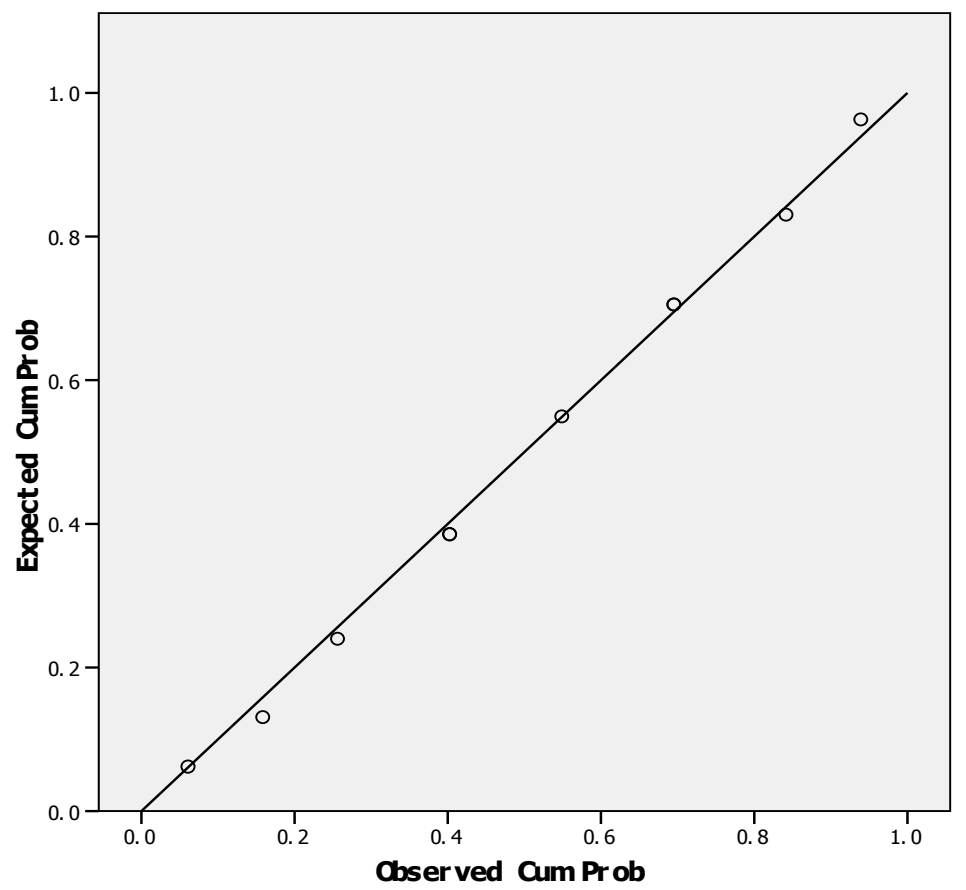

Fig. 1-3. The normal distribution P-P plot of sulcotrione coin residue.

Figure 1-3 shows a plot of the distribution of the sulcotrione residual levels in the 10 corn samples against the expected normal probability distribution. The scatter graph shows an approximately straight line. So the test data follows a normal distribution. The arithmetic average of the sulcotrione soil residual levels in Quzhou and Dongyang was $0.06 \pm 0.02$ $\mathrm{mg} / \mathrm{kg}$.

\subsection{Human serum tyrosine level}

We had 145 valid blood samples collected from the sulcotrione exposed population and the control population. The serum tyrosine concentration in the sulcotrione exposed population was $94.14 \pm 19.67 \mathrm{nmol} / \mathrm{ml}$ for males $(\mathrm{N}=37)$ and $98.85 \pm 21.66 \mathrm{nmol} / \mathrm{ml}$ for females $(\mathrm{N}=$ 31 ). In the control population, the serum tyrosine concentration was $97.60 \pm 16.27 \mathrm{nmol} / \mathrm{ml}$ for males $(\mathrm{N}=35)$ and $100.2 \pm 18.40 \mathrm{nmol} / \mathrm{ml}$ for females $(\mathrm{N}=35)$. In laboratory staff who had contact sulcotrione, the serum tyrosine concentration was $82.02 \pm 23.26 \mathrm{nmol} / \mathrm{ml}$ (male and female, $\mathrm{N}=7$ ), compared to $90.36 \pm 19.27 \mathrm{nmol} / \mathrm{ml}$ (male and female, $\mathrm{N}=10$ ) for laboratory staff who did not have contact with sulcotrione. 


\subsection{The test of rats}

exposed to sulcotrione for 28 days to determine the time and response relationship between blood sulcotrione and tyrosine and main toxic response.

\subsubsection{The general situation of experimental rats}

During the experiment, the body weight, diet, hair, activity in each dose group showed no significant difference from the control group.

\subsubsection{The exposed rat liver, kidneys, adrenal glands and body weight ratio and pathological observations.}

3.3.2.1 The exposed rat liver, kidney and adrenal gland and body weight ratios.

\begin{tabular}{|c|c|c|c|c|}
\hline \multirow{2}{*}{ sex } & group & Liver/body & Kidneys/body & $\begin{array}{c}\text { Adrenal } \\
\text { glands/body }\end{array}$ \\
\hline \multirow{4}{*}{ female } & control & 2.98 & 0.69 & 0.03 \\
\cline { 2 - 5 } & Low-dose & 2.97 & 0.68 & 0.03 \\
\cline { 2 - 5 } & Medium-dose & 2.90 & 0.66 & 0.03 \\
\cline { 2 - 5 } & High-dose & 2.98 & 0.67 & 0.03 \\
\hline \multirow{3}{*}{ male } & control & 3.14 & 0.70 & 0.01 \\
\cline { 2 - 5 } & Low dose & 2.98 & 0.68 & 0.01 \\
\cline { 2 - 5 } & Medium dose & 3.00 & 0.71 & 0.02 \\
\cline { 2 - 5 } & High dose & 3.08 & 0.71 & 0.02 \\
\hline
\end{tabular}

All have $\mathrm{p}>0.05$ in the comparison with the control group.

Table 2-1. The exposed rat liver, kidney and adrenal gland and body weight ratios.

Table 2-1 indicates that in both male and female rats, the liver, kidneys and adrenal glands and the body ratio are not significantly different than those of the controls.

\subsubsection{Pathological observations on the liver, kidney and adrenal gland in each dose} group.

Although there were a few rats in each dose group that showed inflammatory lesions in the liver and kidneys, there was no significant difference in comparison with the control group.

\subsubsection{The serum tyrosine level changes in the male and female rats in each dose group after different exposure times.}

At different sulcotrione exposure times, the rat serum tyrosine levels in the medium- and high-dose groups were significantly higher than those in the low-dose and control groups. The serum tyrosine levels in the high-dose group were significantly higher than those in the medium-dose group. The serum tyrosine concentrations[figure3-1] in the medium- and high-dose groups increased with prolonged exposure before day 21 (the absorption phase). Then they remained relatively stable (the stable phase). After day 28, when exposure ceased, they began to decrease until day 42 (14 days of no exposure). However, they were still higher than the levels of the control at day 42 . There were no significant differences in serum tyrosine levels between male and female rats. 


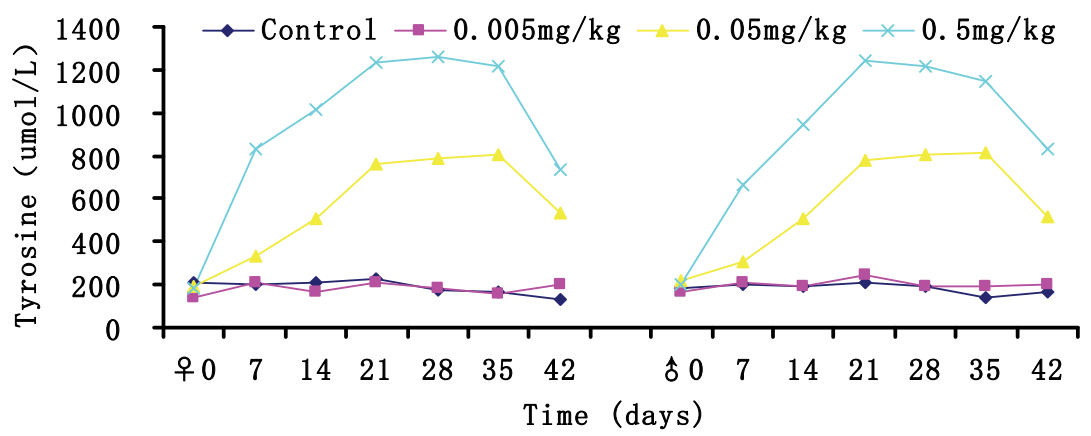

Fig. 3-1. The serum tyrosine level changes in the male and female rats in each dose group after different exposure times.

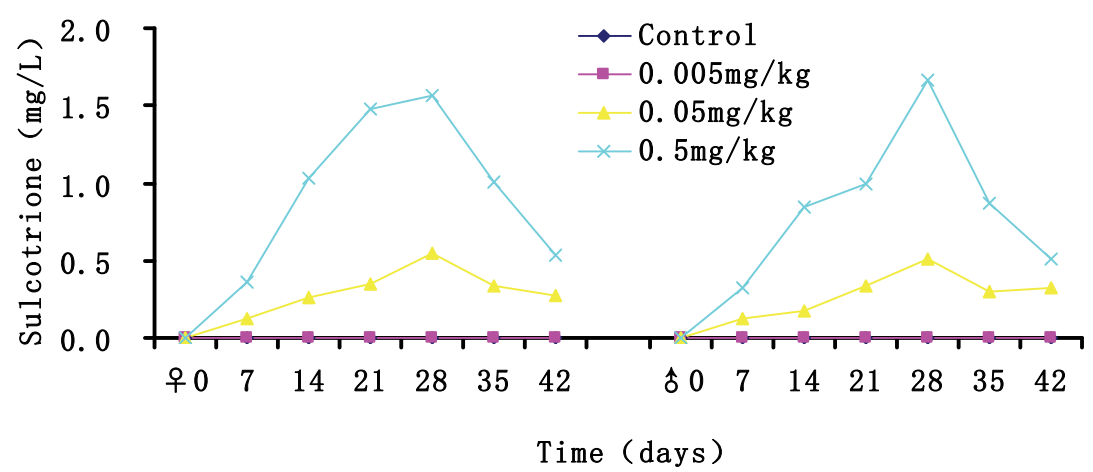

Fig. 3-2. The serum sulcotrione level changes in the male and female rats in each dose group after different exposure times.

The serum sulcotrione in the low-dose group was similar to that in the control group. The serum sulcotrione levels in the males and females were both below the detection limit. The serum sulcotrione was detected in the medium- and high-dose groups, and its level increased with increasing exposure over time and had a statistically significant difference from control. The serum sulcotrione concentration in the medium- and high-dose groups increased with prolonged exposure[figure3-2], but decreased significantly after no exposure. There were no significant differences between male and female rats.

Although there existed individual statistical differences in blood glucose levels between the dose groups and the controls, the values of the changes were all within the normal range [figure3-3]. There were no significant differences in blood glucose changes between male and female rats. 


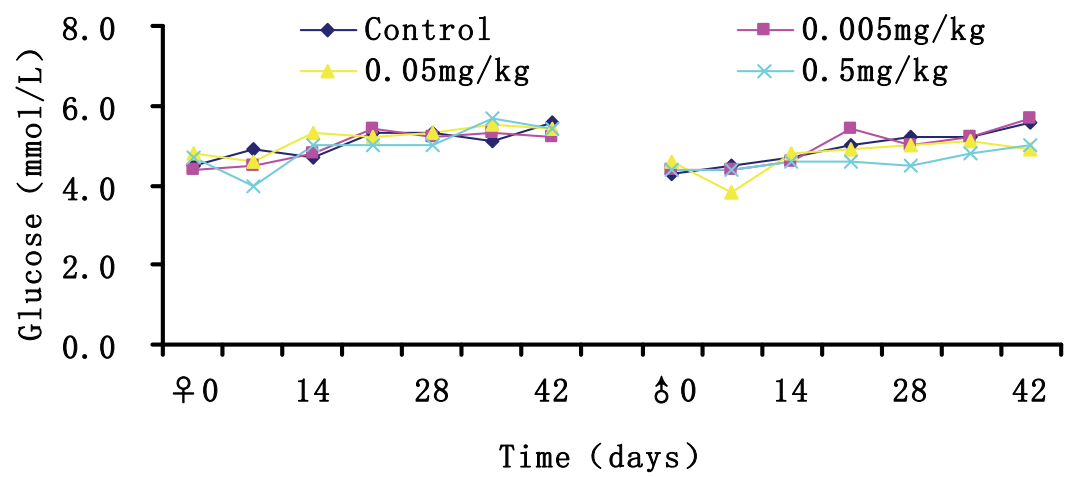

Fig. 3-3. The blood glucose level changes in the male and female rats in each dose group after different exposure times.

\subsubsection{The relationship between serum sulcotrione and tyrosine}

The scatter plots show that between day 7 and 28 of exposure, the serum tyrosine levels increased with the increasing serum sulcotrione in the medium- and high-dose groups. They show a dose-response relationship. There was a positive correlation [figure3-4,figure35]between serum sulcotrione and tyrosine that was statistically significant $(P<0.01)$. This correlation is stronger in the female rats than in the males.

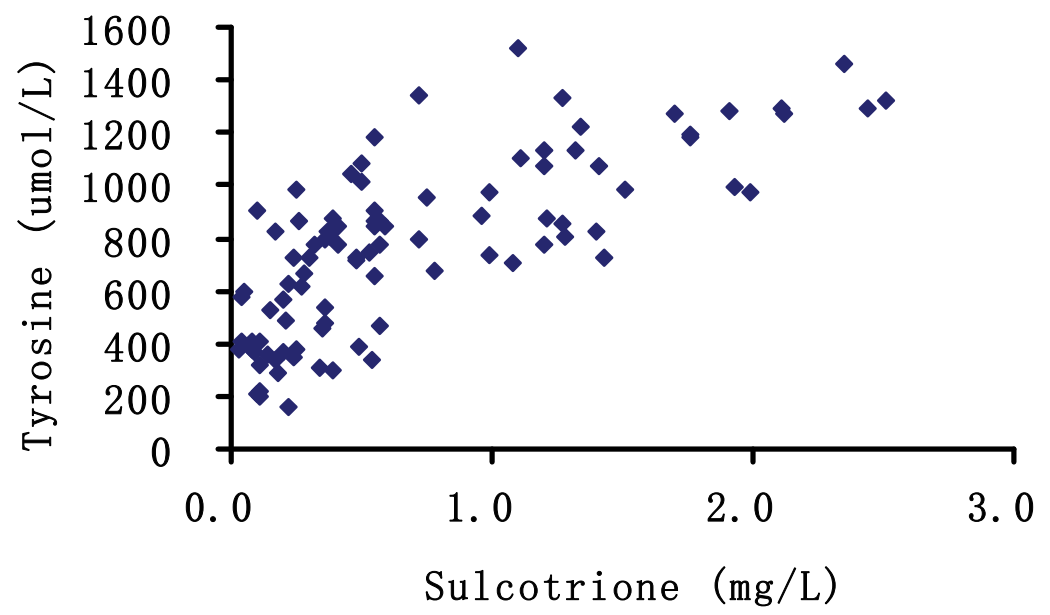

Fig. 3-4. The correlation between serum sulcotrione and tyrosine in female rats from the medium- and high-dose groups. 


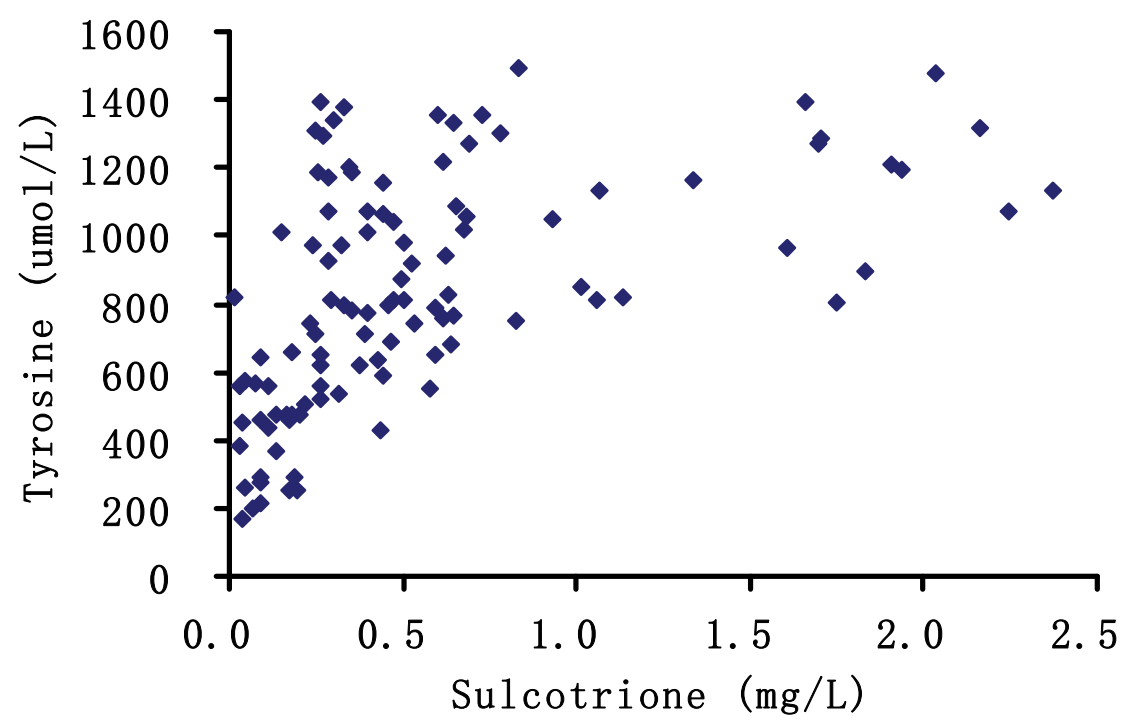

Fig. 3-5. The correlation between serum sulcotrione and tyrosine in male rats from the medium- and high-dose groups.

\subsection{Effects of sulcotrione on hepatic enzymes involved in tyrosine catabolism, tyrosinemia, and blood glucose in rat. \\ 3.4.1 Physical observation and mass gain}

No sign of toxicity was observed in sulcotrione-treated animals until end of experiment and there were no changes in mass gain between controls and sulcotrione-treated animals (date not shown).

\subsubsection{Effects of sulcotrione on hepatic enzymes involved in tyrosine catabolism}

The activities of the hepatic enzymes TAT, HPPD and HGO examined after 90 days were shown at table 4-1. The activity of HPPD was dramatically reduced in liver cytosol by $90 \%$, $92 \%$ and $95 \%$ at doses of $0.1,0.5$ and $5 \mathrm{mg} / \mathrm{kg} /$ day when compared with controls. Significantly higher activity of hepatic TAT $(43 \%, 50 \%, 94 \%)$, the rate limiting enzyme in tyrosine catabolism, was evident in sulcotrione-treated rats at each dose as compared to controls. In contrast, the activity of hepatic $\mathrm{HGO}$ at $5 \mathrm{mg} / \mathrm{kg} /$ day was significantly decreased by $40 \%$ when compared to that of controls, and was not altered at 0.1 and 0.5 $\mathrm{mg} / \mathrm{kg} /$ day.

\subsubsection{Effects of sulcotrione on Serum epinephrine and tyrosine}

Administration of sulcotrione to rats at each dose level for 90 days induced tyrosinemia in rats. There were approximately $717 \%, 817 \%$, and $930 \%$ increases in serum tyrosine levels at $0.1,0.5$ and $5 \mathrm{mg} / \mathrm{kg} /$ day when compared to those of controls (Figure 4-2) and had no effect on their serum epinephrine concentration when compared to the control group (Figure 4-3). 


\begin{tabular}{cccc}
\hline & \multicolumn{3}{c}{ Enzyme activity } \\
\cline { 2 - 4 } $\begin{array}{c}\text { Dose } \\
(\mathrm{mg} / \mathrm{kg})\end{array}$ & $\begin{array}{c}\text { Tyrosine aminotransferase (TAT) } \\
\text { (nmol 4-hydroxyphenylpyruvate } \\
\text { formed/min/mg protein) }\end{array}$ & $\begin{array}{c}\text { 4-Hydroxyphenylpyruvate } \\
\text { dioxygenase (HPPD) } \\
\left(\mu 1 \mathrm{O}_{2} \text { consumed/min/mg }\right. \\
\text { protein) }\end{array}$ & $\begin{array}{c}\text { Homogentisic acid } \\
\text { oxidase }(\mathrm{HGO}) \\
\left(\mu 1 \mathrm{O}_{2}\right.\end{array}$ \\
\hline 0 & $15.15 \pm 1.22$ & $1.55 \pm 0.07$ & $\begin{array}{c}\text { consumed/min/mg } \\
\text { protein })\end{array}$ \\
\hline 0.1 & $19.69 \pm 0.44^{\mathrm{b}}$ & $0.16 \pm 0.02^{\mathrm{b}}$ & $1.14 \pm 0.06$ \\
0.5 & $24.53 \pm 0.73^{\mathrm{b}}$ & $0.12 \pm 0.05 \mathrm{~b}$ & $1.20 \pm 0.05$ \\
5 & $30.49 \pm 0.61^{\mathrm{b}}$ & $0.08 \pm 0.03 \mathrm{~b}$ & $0.96 \pm 0.03$ \\
\hline
\end{tabular}

a Values are mean \pm SE with at least six animals at each dose level.

b $P<0.001$ compared to controls.

Table 4-1. Effects of sulcotrione on hepatic enzymes involved in tyrosine catabolism

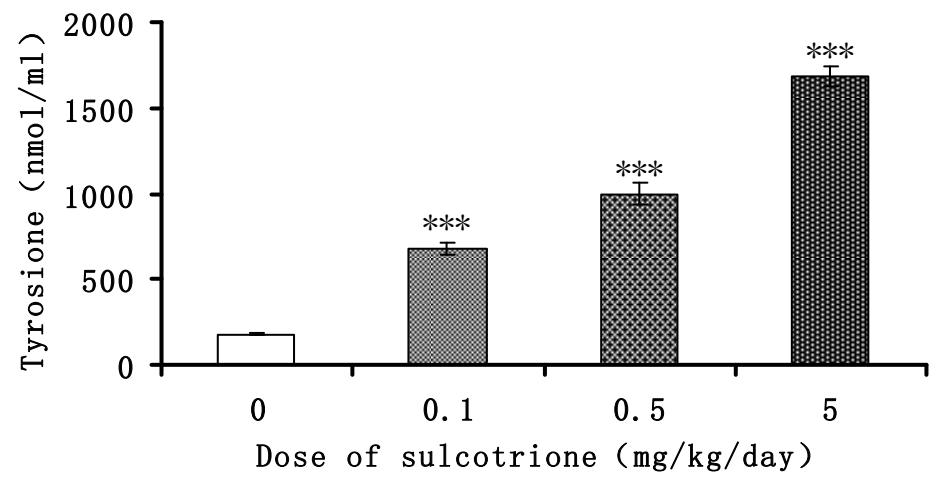

Values are mean $\pm \mathrm{SE}$ with at least six animals at each dose level, ${ }^{* * *} P<0.001$ compared to controls.

Fig. 4-2. Effects of sulcotrione on Serum tyrosine

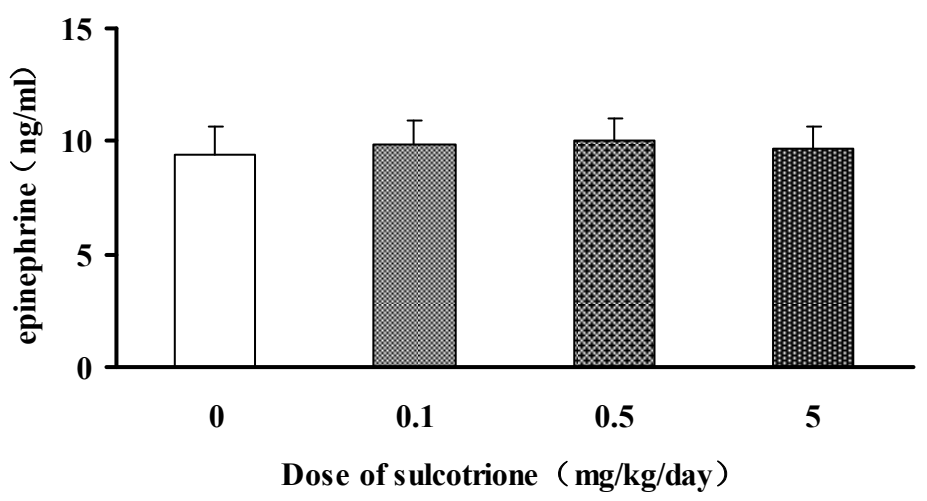

Values are mean \pm SE with at least six animals at each dose level.

Fig. 4-3. Effects of sulcotrione on Serum epinephrine 


\subsubsection{Effects of sulcotrione on Fasting blood glucose and Oral glucose tolerance}

Fasting blood glucose concentration and oral glucose tolerance concentration at each dose in the time as shown at Figure 4-5, and there were not significantly different between controls and sulcotrione-treated animals.

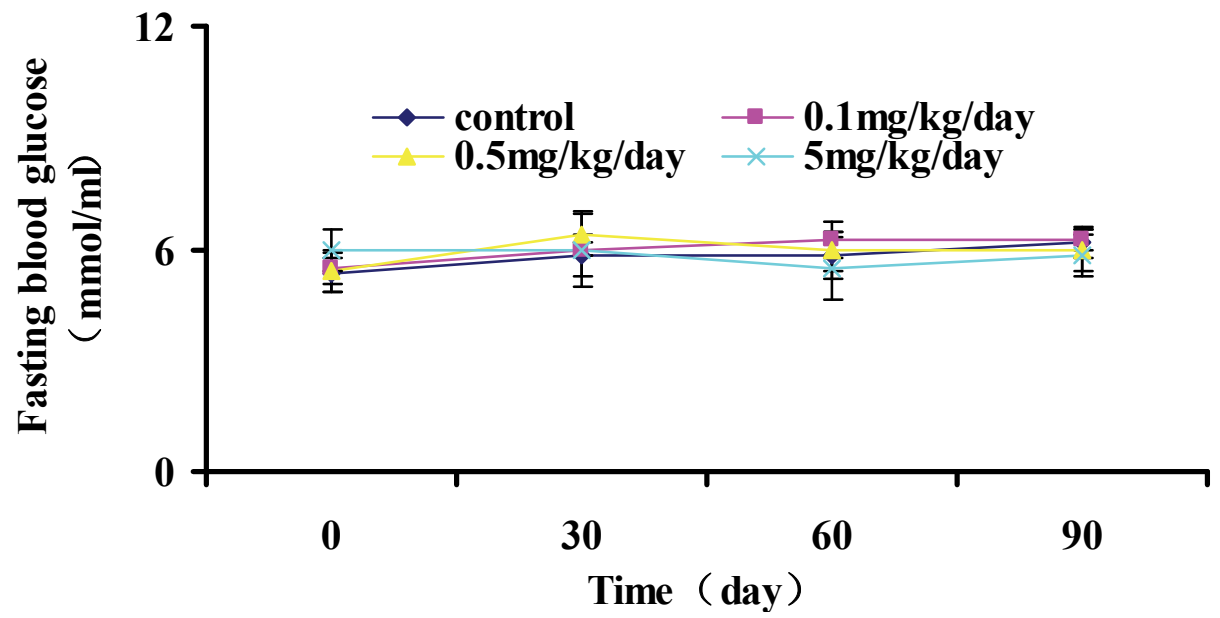

Values are mean \pm SE with at least six animals at each dose level.

Fig. 4-4. Effects of sulcotrione on Fasting blood glucose

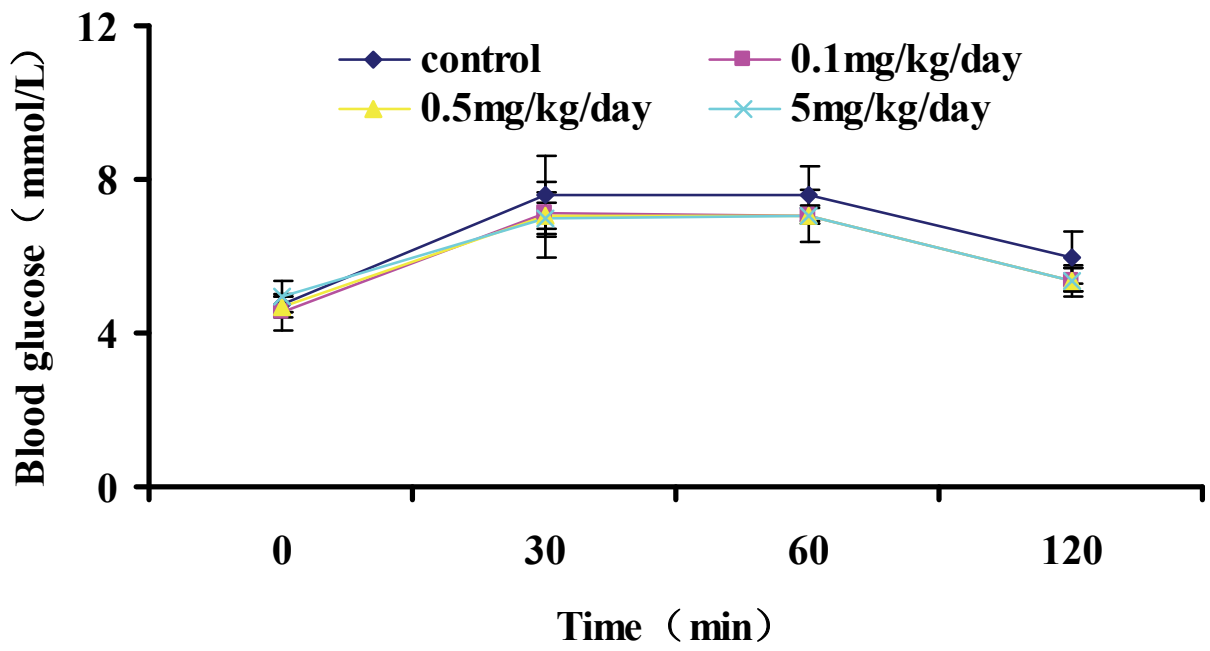

Values are mean \pm SE with at least six animals at each dose level.

Fig. 4-5. Effects of sulcotrione on Oral glucose tolerance 


\subsubsection{Effects of sulcotrione on Serum tyrosine}

Corneal lesions were observed in a few rats given sulcotrione at $5 \mathrm{mg} / \mathrm{kg} /$ day administration of sulcotrione for 90 days. The sulcotrione-treated rats showed corneal lesions that varied in severity from partial or hazy opacity to complete opacity, with edema and neovascularization evident in the more extensive lesions [Fig.4-6].
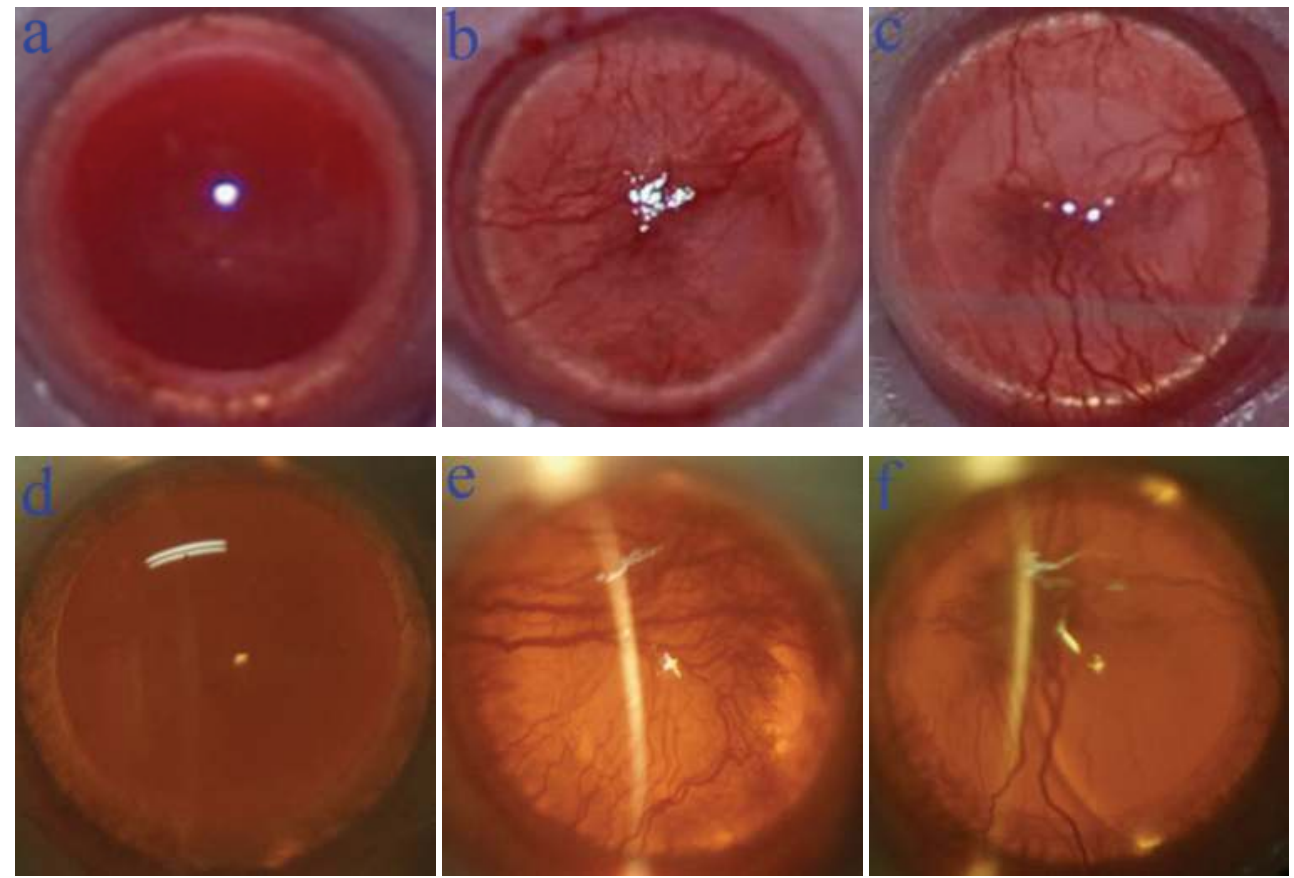

Fig. 4-6. Digital and slit lamp microscope photographs of normal and diseased rat eyes (5 $\mathrm{mg} / \mathrm{kg} /$ day) after oral administration of sulcotrione for 90 days. a and d normal control rat cornea shows a uniformly bright surface and the pupil is clearly visible. $b$ and e opacities over nearly the entire corneal surface and with neovascularization. $\mathrm{c}$ and f opacity of the corneal surface giving it a roughened appearance and edema of the stroma are maximal at the center and less so.

\section{Discussion}

The half-life of soil sulcotrione is up to 122 days [11-14]. The factors that affect the degradation of soil sulcotrione include soil $\mathrm{pH}$, temperature, humidity and soil type. Sulcotrione has a relative shorter residence time in alkaline soil due to the very weak adsorption capacity of alkaline soil for sulcotrione [11]. The degradation rate is very slow in soil with low $\mathrm{pH}$. Red to yellow soil is the main soil type and soil source in Zhejiang Province, located in the western Zhejiang Jinhua and Quzhou area. Cinnamonic soil is mainly located in the northern Zhejiang Jiaxing area. Red to yellow soil belongs to acidic soil, while cinnamonic soil belongs to neutral to slightly alkaline soil. Sulcotrione will be 
degraded quickly in soil in which microorganisms grow well. In low temperature and high humidity conditions, the soil adsorption of sulcotrione will increase and the degradation will decrease [15]. The soil adsorption capacity is correlated with soil particle size and organic matter content. Soil containing more organic content has greater herbicide adsorption capacity. Wilson and Foy indicated that the adsorption of sulcotrione was correlated with the organic content in the soil [16]. Sandy loam and clay has the strongest adsorption capacity for sulcotrione, followed by sandy clay and sandy soil. After adsorption by soil, sulcotrione is gradually released and does not disappear easily [17].

The sulcotrione soil residual level is affected by many factors. This investigation of the current status of sulcotrione soil residual levels aims to provide basic data for use in effective methods of mitigating the environmental damage caused by residual herbicide in the soil during the wide used of the new herbicide.

HPPD is the target molecule of sulcotrione. Its activity can directly reflect the inhibition strength of sulcotrione. At present, the enzymatic activity of HPPD is indirectly and quantitatively measured by the tissue oxygen consumption method, which is somewhat unstable. In this study, the detection of the serum tyrosine level can indirectly reflect the inhibitive activity of sulcotrione on HPPD. Lock, et al. [9] reported that the rat serum tyrosine concentration could be as much as 10 times the normal value after 24 hours when rats were given the sulcotrione analogue 2-nitro-4-trifluoromethylbenzoyl)-1,3cyclohexanedione (NTBC) in a single dose of $0.5 \mathrm{mg} / \mathrm{kg}$. It also caused cornea damage. In this study, corneal opacity in individual rats was also found, which might be related to the accumulation of excessive tyrosine in the anterior chamber of eye. There are three metabolic pathways for tyrosine in mammals: 1) Tyrosine is transferred into 4-hydroxyphenylpyruvic acid (HPPA), catabolized by tyrosine transaminase. It becomes homogentisate after decarboxylation, which is then broken down into acetyl acetate coenzyme A and fumaric acid for the TCA cycle. 2) Tyrosine, catabolized by tyrosine hydroxylase, is transferred into 3,4-dihydroxy phenylalanine (L-DOPA), which is then catabolized by DOPA decarboxylase and converted into dopamine. Dopamine forms norepinephrine after hydroxylation of a carbon atom by dopamine $\beta$ hydroxylase. Finally, epinephrine is formed after methylation of norepinephrine. 3) Tyrosine, catabolized by tyrosine hydroxylase, is transferred into LDOPA, which is then converted into dopaquinone by tyrosinase. The dopaquinone then spontaneously forms melanin. HPPD can transform HPPA into homogentisate by adding oxygen. The transformation from HPPA into homogentisate is inhibited by sulcotrione because it represses the activity of HPPD. Thus, sulcotrione inhibits the first metabolic pathway of tyrosine and induces the accumulation of serum tyrosine in the body, which constitutes the toxicity pathway of sulcotrione. Within the dosages used in this study, the tyrosine level is not high enough to affect blood glucose metabolism through the secondary pathway; or perhaps, even if all tyrosine is metabolized through the secondary pathway, it may still not be enough to affect glucose metabolism.

The results suggest that the metabolism of sulcotrione, although very fast, still has a sustainable impact on tyrosine. With the decreasing sulcotrione burden in the body, the repressed HPPD may be reactivated and the tyrosine metabolic pathway gradually becomes normal. This hypothesis is consistent with the results reported by Ellis et al. [18], who found that NTBC was a potentially reversible inhibitor of HPPD. 


\section{References}

[1] Shaoquan Su, HPPD - New Target for Herbicide Development[J]. PESTICIDES, 2000, 39(5): 4-7

[2] Sehng Guo, Fuming Yang, Lin Zhang, The synthesis of herbicide sulcotrione [J]. PESTICIDES, 2001, 40(7): 20-22

[3] Zhizhong Sun, Wenyi Chu, Yanjun Hou, HPLC determination of sulcotrione[J]. JOURNAL OF NATURAL SCIENCE OF HEILONGJIANG UNIVERSITY, 2004, 21: 108-109

[4] JIN F, wu NX. Determination of sulcotrione in serum by HPLC[J]. Occup Health, 2008, 24(9): 843-844.

[5] Tang AG. Rapid high performance liquid ehromatography for determination of phenylalanine and tyrosine in serum[J]. Bull Hunan Med Univ, 2000, 25(2): 209212.

[6] Mo XM, Tang AG. Determination of phenylalanine and tyrosine in finger blood by highperformance liquid chromatography with ultraviolet detection[J]. Chin J Birth Health Heredity, 2005, 13(3): 29-30.

[7] Zhang D, Chen H. RP-HPLC determination of glycyl-L-tyrosine and its related substance[J]. Chin J Pharm Anal, 2006, 26(5): 664-666.

[8] Hall MG, Wilks MF, Provan WM, Eksborg S, Lumholtz B. Pharmaeokineties and pharmacodynamics of NTBC(2-(2-nitro-4-fluoromethylbenzoy1)-1,3cyclohexanedione) and mesotrione, inhibitors of 4-hydroxyphenyl pyruvate dioxygenase(HPPD)following a single dose to health male volunteers[J]. J Clin Pharmacol, 2001, 52(2): 169-177.

[9] Lock EA, Gaskin P, Ellis MK, Provan WM, Robinson M, Smith LL, et a1. Tissue distribution of 2-(2-nitro-4-trifluoromethylbenzoy1)cyclohexane-1-3-dione(NTBC): effect on enzymes involved in tyrosine catabolism andrelevance to ocular toxicity in the rat[J]. Toxieol Appl Pharmacol, 1996, 141(2): 439-447.

[10] Walker A, Brown PA. The relative persistence in soil of five acetanilide herbicides [J]. Bull Environ Contam Toxicol, 1985, 34: 143-149

[11] Allen R, Walker A. The influence of soil properties on the rates of degradation of metamitron, metazachlor and metribuzin[J]. Pestic Sci, 1987, 18: 95-111

[12] Rouchaud J, Neus O, Callens D et al. soil persistence and mobility in summer maize and winter wheat crops [J]. Weed Res, 1998, 38:361-371

[13] Baer U, Calvet R. Fate of soil applied herbicides: experimental data and prediction of dissipation kinetics [J]. J Environ Qual, 1999, 28: 1765-1777

[14] Beulke S, Malkomes HP. Effects of the herbicides metazachlor and dinoterb on the soil microflora and the degradation and sorption of metazachl or under different environmental conditions[J]. Biol Fertil Soils, 2001, 33:467-471

[15] Laure Mamy, Enrique Barriuso, Benott Gabrielle. Environmental fate of herbicides trifluralin, metazachlor, metamitron and sulcotrione compared with that of glyphosate, a substitutebroad spectrum herbicide for different glyphosateresistant crops [J]. Pest Manag Sci, 2005, 61: 905-916

[16] Wilson S, Foy C. Influence of various soil properties on the absorption anddesorption of ICIA-0051 in five soils [J]. Weed Technol, 1992, 6: 583-586.

[17] Moshier LJ, Penner D. Factors influencing microbial degradation of 14C-glyphosate to 14CO2 in soil [J]. Weed Sci, 1978, 26: 686-691 
[18] Ellis MK, Whitfield AC, Gowans LA, Auton TR, Pmvan WM, et al. Inhibition of 4hydroxyphenylpyruvate dioxygenase by 2-(2-nitro-4trifluommethylbenzoy1)cyclohexanel, 3-dione and 2-(2-nitro-4-methanesulphonyl benzoy1)-cy-elohexanel, 3-dione[J]. Toxicol appl Pharmacol, 1995, 133(1): 12-19. 


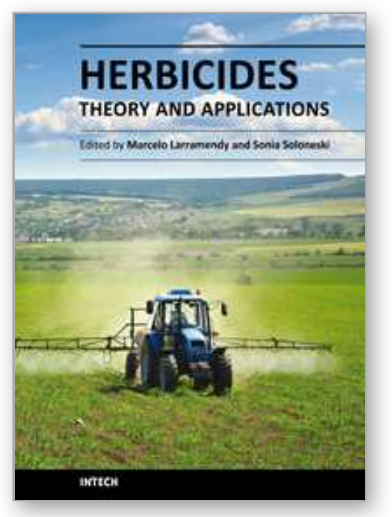

\author{
Herbicides, Theory and Applications \\ Edited by Prof. Marcelo Larramendy
}

ISBN 978-953-307-975-2

Hard cover, 610 pages

Publisher InTech

Published online 08, January, 2011

Published in print edition January, 2011

The content selected in Herbicides, Theory and Applications is intended to provide researchers, producers and consumers of herbicides an overview of the latest scientific achievements. Although we are dealing with many diverse and different topics, we have tried to compile this "raw material" into three major sections in search of clarity and order - Weed Control and Crop Management, Analytical Techniques of Herbicide Detection and Herbicide Toxicity and Further Applications. The editors hope that this book will continue to meet the expectations and needs of all interested in the methodology of use of herbicides, weed control as well as problems related to its use, abuse and misuse.

\title{
How to reference
}

In order to correctly reference this scholarly work, feel free to copy and paste the following:

Nanxiang Wu (2011). Herbicide Sulcotrione, Herbicides, Theory and Applications, Prof. Marcelo Larramendy (Ed.), ISBN: 978-953-307-975-2, InTech, Available from: http://www.intechopen.com/books/herbicides-theoryand-applications/herbicide-sulcotrione

\section{INTECH}

open science | open minds

\section{InTech Europe}

University Campus STeP Ri

Slavka Krautzeka 83/A

51000 Rijeka, Croatia

Phone: +385 (51) 770447

Fax: +385 (51) 686166

www.intechopen.com

\section{InTech China}

Unit 405, Office Block, Hotel Equatorial Shanghai

No.65, Yan An Road (West), Shanghai, 200040, China

中国上海市延安西路65号上海国际贵都大饭店办公楼 405 单元

Phone: +86-21-62489820

Fax: $+86-21-62489821$ 
(C) 2011 The Author(s). Licensee IntechOpen. This chapter is distributed under the terms of the Creative Commons Attribution-NonCommercialShareAlike-3.0 License, which permits use, distribution and reproduction for non-commercial purposes, provided the original is properly cited and derivative works building on this content are distributed under the same license. 